\title{
Levantamento das distribuições de temperatura em leito de arroz com casca durante o tratamento térmico por micro-ondas
}

\author{
Determination of the temperature distributions in a paddy rice bed \\ during heat treatment with microwaves
}

\section{Autores | Authors}

$\triangle$ Michele Nehemy BERTELI

Instituto de Tecnologia de Alimentos (ITAL)

Grupo de Engenharia de Processos (GEPC)

Av. Brasil, 2880 - Jardim Chapadão CEP: $13070-178$

Campinas/SP - Brasil e-mail: berteli@ital.sp.gov.br

Taileah Leite NOGUEIRA

Universidade de São Paulo (USP)

Faculdade de Engenharia de Alimentos Pirassununga/SP - Brasil e-mail: taileah@usp.br

\section{Antonio MARSAIOLI JUNIOR}

Instituto de Tecnologia de Alimentos (ITAL) Grupo de Engenharia de Processos (GEPC)

Campinas/SP - Brasil e-mail: tonymars@ital.sp.gov.br

$\square$ Autor Correspondente / Corresponding Author

Recebido: Ago. 19, 2014

Aprovado: Jan. 20, 2015
Resumo

Durante o aquecimento dielétrico, a distribuição do campo elétrico dentro de uma cavidade ocorre a partir de uma propagação multimodal, ocasionada por inúmeras reflexões das micro-ondas provenientes das paredes metálicas da cavidade. Essas reflexões múltiplas geram regiões de maior e menor intensidade de campo elétrico no espaço e, por consequência, um aquecimento não uniforme no produto. O objetivo deste estudo foi o levantamento dos valores de temperaturas obtidos durante o tratamento térmico por micro-ondas em um leito de arroz em casca úmido posicionado em um porta-amostra, dentro de uma cavidade que apresenta uma geometria prismática de seção sextavada. A cavidade foi desenhada para, operando em frequência de $5,8 \mathrm{GHz}$ e potência máxima de $700 \mathrm{~W}$, minimizar a heterogeneidade de distribuição das intensidades do campo elétrico, gerando um aquecimento mais uniforme do produto. Verificou-se que as diferenças de temperatura registradas pelos sensores no leito de arroz foram de $3,2{ }^{\circ} \mathrm{C}$ a $12,5^{\circ} \mathrm{C}$ durante os tratamentos térmicos estudados. Não foi observado um comportamento padrão que indicasse uma região de maior aquecimento em relação à altura e direção radial do leito de arroz durante os tratamentos térmicos por micro-ondas

Palavras-chaves: Micro-ondas; Campo elétrico; Diferença de temperatura; Arroz em casca.

\section{Summary}

During dielectric heating the electric field distribution inside a cavity occurs as from a multimodal propagation caused by countless reflections coming from the metallic walls of the cavity. These multiple reflections generate regions of greater and lesser intensities of the electric field in the space and, as a consequence, non-uniform product heating. The objective of this study was to determine the temperatures obtained during the microwave treatment of a moist paddy rice bed placed in a sample container inside the application cavity. The cavity had a prismatic geometry with a hexagonal section and was designed such that when operating at $700 \mathrm{~W}$ and $5.8 \mathrm{GHz}$ it minimized the heterogeneity of the distribution of the electric field intensities, so as to generate a more uniform product heating. The differences registered by the sensors in the paddy rice bed ranged from 3.2 to $12.5^{\circ} \mathrm{C}$ during the thermal treatments studied. No standard behaviour was observed indicating a region with more intense heating with respect to the height and radial directions inside the moist paddy rice bed in the sample container in the prismatic cavity.

Key words: Microwaves; Electric field; Temperature difference; Paddy rice. 


\section{Introdução}

A tecnologia de micro-ondas pode desempenhar um papel relevante na indústria de alimentos, gerando um rápido aquecimento dos materiais submetidos a esse tipo de energia. Como consequência, os processos que usam o aquecimento dielétrico frequentemente apresentam vantagens na qualidade dos produtos finais sem comprometer significativamente as características nutricionais, funcionais e sensoriais dos alimentos, as quais constituem demandas cada vez maiores do mercado consumidor. Outras vantagens dessa tecnologia inovadora e de grande potencial estão relacionadas a uma melhoria considerável no rendimento econômico, com tempos de processamento mais curtos, menores espaços físicos requeridos e redução do uso de insumos, favorecendo ainda mais a relação benefício/custo. Além dessas vantagens, as micro-ondas são já altamente eficientes desde o momento de sua geração.

Os materiais dielétricos têm em comum a capacidade de armazenar energia elétrica. Existem vários mecanismos em escala molecular e atômica para a conversão de energia de micro-ondas em energia térmica (BUFFLER, 1992).

As propriedades dielétricas dos materiais medem a interação entre o material e o campo elétrico aplicado, em temperatura e frequência específicas. Os parâmetros que caracterizam os materiais dielétricos (NELSON e DATTA, 2001) são:

- Permissividade (ou constante dielétrica) relativa $\varepsilon_{\mathrm{r}}^{\prime}$ : Avalia a capacidade de um material armazenar energia elétrica, de maneira reversível;

- Fator (ou constante) de perda relativa $\varepsilon_{r}$ ": Parâmetro dado pelas propriedades elétricas de dissipação de energia elétrica sob a forma de calor, nesse caso, de maneira irreversível;

- Tangente de perdas: O coeficiente dos dois valores

$\operatorname{tang}=\varepsilon_{\mathrm{r}}{ }^{\prime \prime} / \varepsilon_{\mathrm{r}}{ }^{\prime}$

A Equação 1 indica a capacidade de o material ser penetrado por um campo elétrico e de dissipar essa energia na forma de calor, ou seja, determina a sua extensão de reflexão e transmissão de energia. As aplicações do aquecimento por micro-ondas na indústria, na sua maioria, são relativas à água (KOMAROV, 2012). A água líquida possui, em comparação com os dielétricos frequentes, os valores mais elevados de $\varepsilon_{r}^{\prime}$ e $\varepsilon_{r}^{\prime}$ " (NELSON e DATTA, 2001; TANG, 2005).

A determinação das propriedades dielétricas é importante para a previsão da taxa de aquecimento do material quando submetido à radiação micro-ondas. É também a partir dos valores de $\varepsilon_{r}^{\prime}$ e $\varepsilon_{r}$ " possível estimar a penetração das micro-ondas $\left(D_{p}\right)$, Equação 2, nos materiais dielétricos, na frequência estudada, e, dessa forma, definir a quantidade e distribuição espacial do material presente na cavidade durante os testes de tratamento térmico por micro-ondas. A profundidade de penetração $D_{p}$ é definida como a distância na qual a potência é atenuada para $1 / e=1 / 2,7183=0,37$, ou $37 \%$ do seu valor na superfície do material (TANG, 2005).

$$
D p=\left(\lambda_{0} \cdot \sqrt{\left.\varepsilon_{r}^{\prime}\right)} /\left(2 \pi \cdot \varepsilon_{r}^{\prime \prime}\right)\right.
$$

sendo $\lambda_{0}$ o comprimento de onda no espaço livre.

É usual a afirmativa de que "as micro-ondas aquecem o material de dentro para fora". Mas, geralmente, isso não ocorre, pois a penetração das ondas é frequentemente menor que a espessura dos alimentos tratados (RISMAN, 2009).

Durante o aquecimento dielétrico, a distribuição do campo elétrico dentro de uma cavidade ocorre a partir de uma propagação multimodal, ocasionada por inúmeras reflexões das micro-ondas provenientes das paredes metálicas da cavidade. Essa característica é um fator indesejado associado ao aquecimento por micro-ondas, pois as reflexões múltiplas geram regiões de maior e de menor intensidade de campo elétrico no espaço, devido ao fenômeno de onda estacionária, resultante da superposição de ondas que se propagam em sentidos opostos. A onda refletida, nos pontos em que chega em fase com a onda incidente, soma-se à mesma e aumenta o valor do campo elétrico; chegando defasada, é subtraída e diminui o campo elétrico. Isso resulta em uma distribuição não uniforme do campo elétrico dentro de uma cavidade e, consequentemente, em um aquecimento não uniforme (BUFFLER, 1992).

O tratamento térmico visando a destruição microbiológica e/ou enzimática por micro-ondas em aplicadores monomodais em sistemas contínuos oferece aquecimento rápido e eficiente e vem sendo bastante pesquisado pela comunidade científica (BOLDOR et al., 2008; CORONEL et al., 2008; LIN e RAMASWAMY, 2011). Entretanto, no tratamento térmico de materiais sólidos, em cavidade multimodo, uma grande dificuldade é justamente a falta de perfis homogêneos de temperatura no produto durante o aquecimento por micro-ondas, uma vez que o padrão de aquecimento pode ser bastante irregular. Vadivambal e Jayas (2010) relacionam a distribuição não uniforme da temperatura do produto como o principal fator limitante para o desenvolvimento de processos industriais de pasteurização por micro-ondas.

Em um estudo desenvolvido por Koskiniemi et al. (2011) foram levantados os perfis de temperatura de vegetais em pedaços durante a pasteurização em um túnel contínuo de micro-ondas na frequência de $915 \mathrm{MHz}$. Os vegetais, brócolis, pimenta vermelha e batata-doce, foram acidificados em solução na proporção de $0,75 \%$ p/p de ácido cítrico e acondicionados em copos de polipropileno, 
com peso líquido de $90 \mathrm{~g}$. Para o levantamento dos perfis de temperatura dos vegetais durante o tratamento térmico por micro-ondas, cinco fibras ópticas foram inseridas em um dos copos em posições distintas, no centro dos vegetais. O tempo de permanência no túnel foi de 4 min, a uma potência incidente de $4 \mathrm{~kW}$. As temperaturas máximas obtidas durante o tratamento térmico nas cinco posições variaram de: batata-doce, de $100,0^{\circ} \mathrm{C}$ a $79,2^{\circ} \mathrm{C}$; pimenta vermelha, de $98,7{ }^{\circ} \mathrm{C}$ a $59,4{ }^{\circ} \mathrm{C}$; brócolis, de $100{ }^{\circ} \mathrm{C}$ a $73,8^{\circ} \mathrm{C}$

O padrão do campo eletromagnético das microondas pode ser configurado a partir da geometria da cavidade adotada no seu projeto, incluindo-se nele o projeto do sistema de guia de ondas. Entretanto, a uniformidade do aquecimento é também influenciada pela geometria, tamanho, propriedades dielétricas e propriedades térmicas dos alimentos (WÄPPLINGRAAHOLT e OHLSSON, 2005).

O objetivo deste estudo é o levantamento dos perfis e diferenças de temperatura em um leito de arroz com casca úmido durante o tratamento térmico por micro-ondas em uma cavidade multi-modo de geometria prismática de seção sextavada.

\section{Material e métodos}

\subsection{Sistema de aplicação de micro-ondas}

No sistema de aplicação de micro-ondas, Figura 1, o equipamento principal é composto por uma cavidade multimodo de micro-ondas de geometria de seção sextavada prismática, para que a heterogeneidade tridimensional das intensidades do campo elétrico na configuração de ondas estacionárias possa ser minimizada, estabelecendo-se de forma dispersa (1), desde que as dimensões das amostras sejam compatíveis com o comprimento de onda na frequência de 5,8 GHz. A cavidade está ligada a um gerador de micro-ondas (5) de 5,8 GHz x 700 W (Gerling Applied Engineering, GA4002A), que transmite as micro-ondas mediante uma guia de onda de seção retangular (6) conectada a um conjunto monitorador de potência de micro-ondas constituído dos seguintes componentes: um circulador de três portas (7), um banho termostático (8), um acoplador duplo (9), dois watímetros (10) para medir potências de micro-ondas transmitidas e refletidas, um sintonizador de impedância de 4 tocos (11), terminando a guia de onda em curva de $90^{\circ}$ até a entrada na tampa superior da cavidade. As leituras pontuais e o registro das temperaturas do produto foram obtidos por meio de um aquisitor (Luma Sense Technologies - Luxtron Fot Lab Kit) de temperatura de fibra óptica (12) conectado a um computador (13).

\subsection{Caracterização do arroz e estimativa da penetração de ondas}

Utilizou-se arroz em casca como material pois o presente estudo serviu como suporte para o desenvolvimento de uma pesquisa paralela na mesma cavidade de micro-ondas que aborda o envelhecimento acelerado, a estabilidade do farelo e a secagem dos grãos de arroz recém-colhidos.

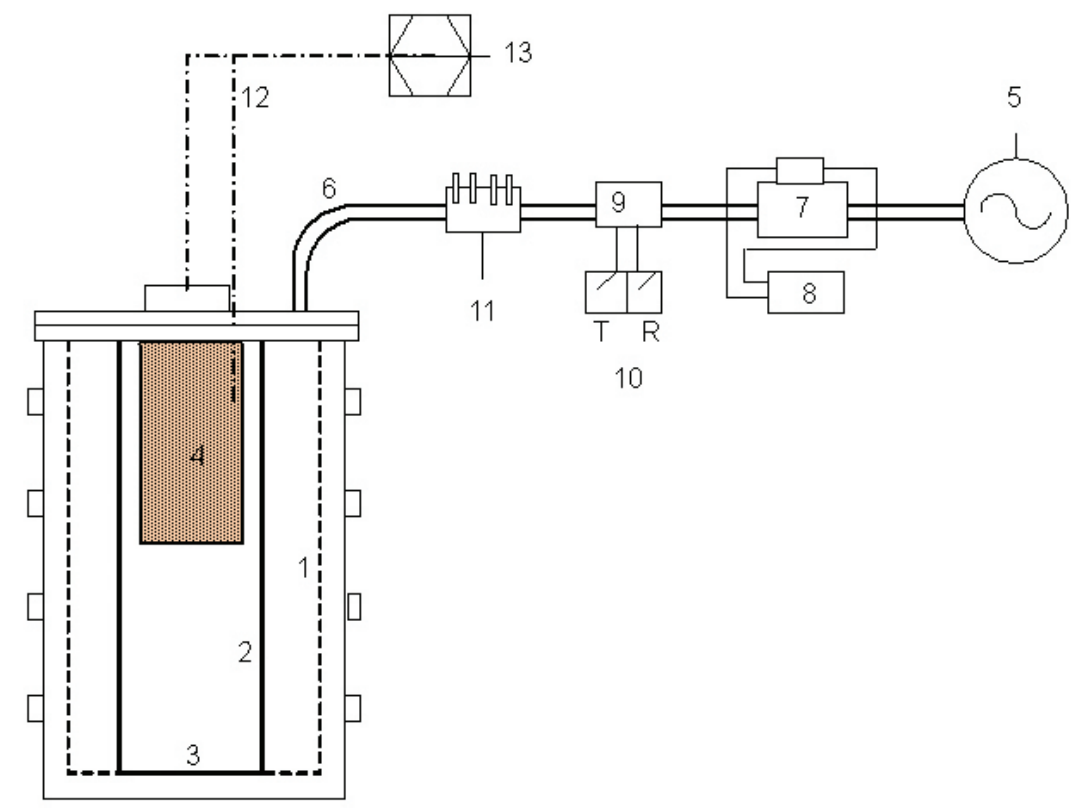

Figura 1. Sistema do aplicador de micro-ondas: (1) cavidade de micro-ondas prismática sextavada; (2) tubo de vidro de borosilicato; (3) suporte; (4) cesto Teflon (porta-amostra); (5) gerador de micro-ondas; (6) guia de onda; (7) circulador de três portas; (8) banho termostático; (9) acoplador duplo; (10) watímetros, T: potência transmitida $\left(P_{T}\right) ; R$ : potência refletida $\left(P_{R}\right)$; $(11)$ sintonizador; $(12)$ fibra óptica; (13) computador. 
Para a realização deste estudo escolheu-se o cultivar IAC 202, que apresenta característica aglutinante e pegajosa após cozimento. No Brasil, a preferência é por um arroz solto e seco, macio e com aroma, que são características de um arroz envelhecido, fator que favorece o estudo de envelhecimento acelerado do arroz. O processo de envelhecimento natural é demorado, podendo durar até 6 meses, e necessita de um armazenamento adequado. $O$ envelhecimento acelerado de grãos de arroz é importante não só para minimizar os custos de processamento devido ao armazenamento prolongado como as perdas decorrentes desse armazenamento (RAYAGURU et al., 2011; ROCHA, 2002).

Realizou-se uma hidratação do arroz para reproduzir a condição de umidade dos grãos recémcolhidos, necessária para o processo de envelhecimento acelerado e estabilização do farelo dos grãos de arroz. Os grãos de arroz com casca foram hidratados com água destilada durante $4 \mathrm{~h}$, a $70^{\circ} \mathrm{C}$, e apresentaram uma umidade final de cerca de $28 \%$ b.u. A umidade dos grãos foi determinada por um analisador de umidade por micro-ondas (Smart 5 - CEM) calibrado segundo o método oficial da AOAC (2006).

A constante dielétrica relativa $\varepsilon_{r}^{\prime}$ e o fator de perda dielétrica relativa $\varepsilon_{\mathrm{r}}^{\prime}$ para os grãos de arroz foram estimados através das Equações 3 e 4, desenvolvidas por Kraszewski e Nelson (1989) e aplicadas para grãos em faixa de frequência de 5 a $5.000 \mathrm{MHz}$ e de teor de umidade de $8 \%$ a $26 \%$. A frequência utilizada foi aproximada para $5 \mathrm{GHz}$ e a umidade inicial para $26 \%$. As propriedades dielétricas foram estimadas para as umidades inicial, antes, e final, após o processo por micro-ondas dos grãos, sendo essa última definida por testes preliminares.

$\varepsilon_{r}^{\prime}=\left(1+\frac{0,504 \cdot U \cdot \rho}{\sqrt{U}+\log f}\right)^{2}$ $\varepsilon_{\mathrm{r}}{ }^{\prime}=0,146 \cdot \rho_{\mathrm{g}}{ }^{2}+0,004615 \cdot \mathrm{U}^{2} \cdot \rho_{\mathrm{g}}{ }^{2}\left(0,32 \cdot \log \mathrm{f}+\frac{1,743}{\log \mathrm{f}}-1\right)$

sendo $\mathrm{U}$ a umidade do grão em base úmida (\%); $\rho_{\mathrm{g}}$ a densidade aparente do arroz $\left(\mathrm{g} / \mathrm{cm}^{3}\right) ; f$ a frequência de micro-ondas ( $\mathrm{MHz}$ ).

A densidade aparente do arroz foi obtida de acordo com o modelo desenvolvido por Nelson e Datta (2001) em função da umidade do grão, Equação 5.

$$
\tilde{n}_{g}=0,6829+0,01422 \cdot U-0,000979 \cdot U^{2}+0,0000153 \cdot U^{3}(5)
$$

A partir dos valores calculados de $\varepsilon_{r}^{\prime}$ e $\varepsilon_{r}^{\prime \prime}$ foi possível estimar a penetração das micro-ondas $\left(D_{p}\right)$, Equação 2, para as umidades inicial, antes, e final, após o processo por micro-ondas do arroz, na frequência de trabalho. Dessa forma foi definida a altura do leito de arroz e sua correspondente massa no porta-amostra durante os tratamentos por micro-ondas, de maneira a permitir a penetração das micro-ondas em todo o leito.

\subsection{Levantamento das temperaturas do leito de arroz}

Para o estudo da evolução da temperatura do leito de arroz durante os tratamentos térmicos por microondas, amostras do produto foram posicionadas dentro de um cesto cilíndrico de malha de fibra de vidro revestido de Teflon de 8,7 cm de diâmetro, fixado a um anel de alumínio de suporte (porta-amostra). Para evitar interferências na distribuição do campo elétrico dentro da cavidade, somente o corpo do cesto não metálico permaneceu dentro da cavidade, por ser de material transparente a micro-ondas. O cesto possuía uma tampa metálica com orifícios de diâmetro previamente calculado, de maneira a permitir a passagem dos sensores de fibra óptica e impedir o vazamento de micro-ondas na frequência de 5,8 GHz. A tampa permaneceu fora da cavidade de microondas, sobre a parte metálica do cesto, fixadas por uma abraçadeira (Figura 2a).
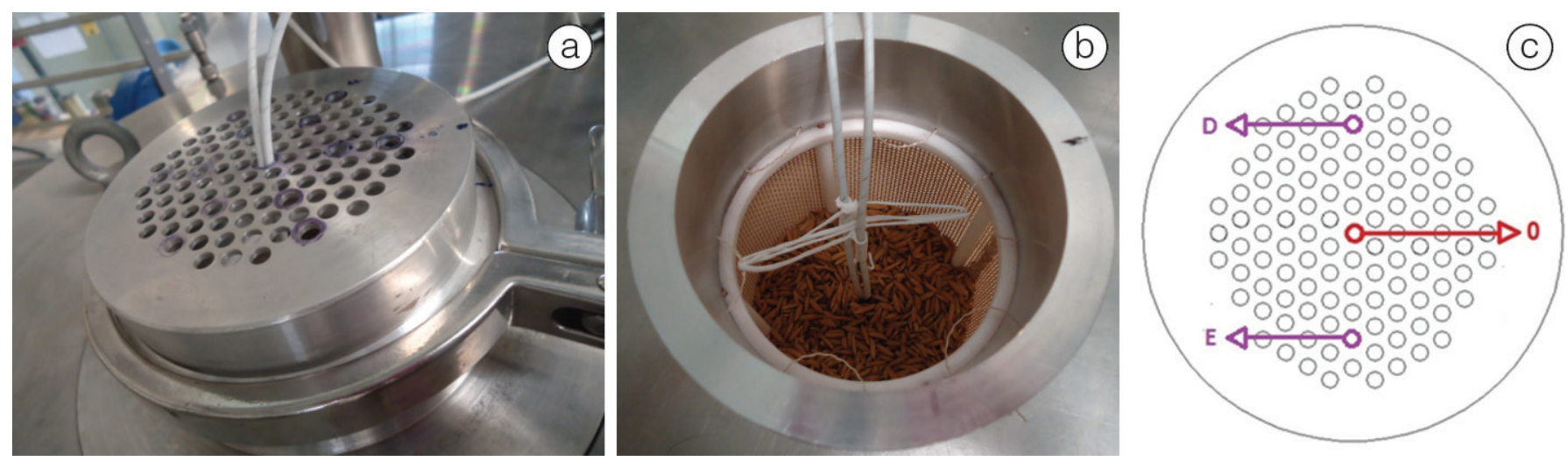

Figura 2. (a): Cesto de Teflon posicionado no interior da cavidade de micro-ondas, sob a tampa perfurada e fixada por uma abraçadeira; (b): Fibras ópticas posicionadas no leito de arroz; (c): Esquema do posicionamento das fibras ópticas em relação à tampa do cesto de Teflon. 
Após o assentamento do cesto na cavidade de micro-ondas, duas fibras ópticas foram posicionadas com a ajuda de fita de Teflon no interior do porta-amostra, para o acompanhamento das temperaturas do leito de arroz durante o tratamento térmico (Figura 2b). A posição das fibras ópticas variou com o tratamento térmico. $\mathrm{O}$ posicionamento dos sensores em relação à tampa do cesto está representado na Figura 2c.

Os pontos E e D (Figura 2c) representam as extremidades esquerda e direita do cesto, respectivamente. Já o ponto 0 representa o centro do cesto e nessa situação o posicionamento das fibras ópticas varia em relação à altura do leito de arroz.

O arroz após a hidratação foi transferido para o porta-amostra em quantidades pré-determinadas, baseadas nos cálculos de penetração de onda, sem qualquer compactação. Os ensaios foram realizados em triplicata.

\section{Resultados e discussão}

\subsection{Estimativa de penetração de onda}

A partir dos valores calculados de $\varepsilon_{r}^{\prime}$ e $\varepsilon_{r}^{\prime \prime}$ foi estimada a penetração das micro-ondas $\left(D_{p}\right)$ no leito de arroz e então definida a quantidade do material no portaamostra durante os tratamentos térmicos por micro-ondas. Os valores calculados de $\varepsilon_{r}^{\prime}, \varepsilon_{r}^{\prime \prime}$ e $D_{p}$ estão apresentados na Tabela 1. A penetração de onda na frequência de $5,8 \mathrm{GHz}$ foi estimada para o início do tratamento térmico em $1,7 \mathrm{~cm}$ e, para o final do tratamento, na menor umidade do grão (16\%), em 3,3 cm. Observa-se, de acordo com os resultados obtidos, que a penetração da onda no leito de arroz é menor quando o grão apresenta umidade maior (26\%), em virtude da maior capacidade de absorção de micro-ondas já em sua superfície.

Como o corpo do cesto de Teflon é formado por material transparente a micro-ondas, a penetração das ondas ocorre por todas as direções. Portanto, a quantidade de material foi definida de maneira a não

Tabela 1. Valores estimados de $\varepsilon_{r}^{\prime}, \varepsilon_{r}^{\prime \prime}$ e $D_{p}$ para o leito de arroz.

\begin{tabular}{cccc}
$\mathbf{U}(\%)$ & $\varepsilon_{\mathbf{r}}^{\prime}$ & $\varepsilon_{\mathrm{r}}^{\prime}$ & $\mathbf{D}_{\mathbf{p}}(\mathbf{c m})$ \\
\hline 26 & 3,87 & 0,92 & 1,8 \\
16 & 2,93 & 0,43 & 3,3 \\
\hline
\end{tabular}

ultrapassar 3,6 cm de altura $(2 \times 1,8 \mathrm{~cm})$ e dessa forma permitir que as micro-ondas atingissem todo o leito da amostra posicionada no cesto, ou seja, pela parte inferior e superior do porta-amostra.

Portanto, para a realização dos ensaios, as quantidades das amostras foram de $100 \mathrm{~g}$ e $200 \mathrm{~g}$, valores que corresponderam, respectivamente, a uma altura do leito de $1,7 \mathrm{~cm}$ e $3,5 \mathrm{~cm}$ no cesto de Teflon.

\subsection{Levantamento das temperaturas do leito de arroz}

As condições dos tratamentos térmicos por microondas aplicados ao leito de arroz estão apresentadas na Tabela 2. Nos ensaios 1, 2 e 3, uma fibra óptica foi posicionada no ponto $E$ e a outra, no ponto $D$, após a adição de $100 \mathrm{~g}$ de arroz no porta-amostra, assegurando-se que os sensores estivessem totalmente imersos no produto. Nos ensaios 4, 5 e 6, a primeira fibra óptica foi posicionada após a colocação de $150 \mathrm{~g}$ de arroz (ponto denominado meio) e a segunda, após 50 gramas adicionais (ponto denominado superfície), totalizando $200 \mathrm{~g}$, ambas no ponto 0 (centro do cesto). Já nos ensaios 7, 8 e 9, a primeira fibra óptica foi posicionada após a adição de $50 \mathrm{~g}$ de arroz (ponto denominado fundo) e a segunda, após a adição de mais $100 \mathrm{~g}$ (ponto denominado meio), ambas no ponto 0 , e então mais $50 \mathrm{~g}$ de arroz foram inseridos, totalizando $200 \mathrm{~g}$ de material inicial. As micro-ondas foram ligadas para o início de cada processo após o posicionamento das duas fibras ópticas na amostra para a leitura das temperaturas.

O término dos experimentos foi estipulado quando uma das fibras ópticas atingisse primeiramente $92^{\circ} \mathrm{C}$. O final do processo correspondeu à obtenção da temperatura usada para a estabilização do farelo de arroz com a inativação da lipase $\left(92^{\circ} \mathrm{C}\right)$, a partir da aplicação de micro-ondas no arroz recém-colhido (ROCHA, 2002).

Em todos os ensaios realizados, a potência refletida indicada pelo watímetro - R (Figura 1) foi inferior a $2 \%$. Desse modo, estima-se que a potência absorvida pelo material foi correspondente à potência transmitida $-P_{T}$.

A Figura 3 apresenta a evolução das temperaturas do arroz e a Figura 4, a evolução das diferenças de temperatura $(\Delta T)$ durante os tratamentos térmicos por micro-ondas registradas pelas fibras ópticas, correspondentes aos ensaios 1, 2 e 3. Esses ensaios

Tabela 2. Condições dos ensaios desenvolvidos durante os tratamentos por micro-ondas, sendo a umidade inicial do arroz $=28 \%$ b.u.; $P_{T}=$ potência transmitida; $\rho_{p}=$ densidade de potência inicial.

\begin{tabular}{|c|c|c|c|c|}
\hline Ensaios & Massa $(g)$ & $P_{T}(W)$ & $\rho_{p}(W / g)$ & $\begin{array}{l}\text { Posição das fibras ópticas } \\
\text { relativa à tampa do cesto }\end{array}$ \\
\hline 1,2 e 3 & 100 & 100 & 1,0 & E e D \\
\hline 4,5 e 6 & 200 & 100 & 0,5 & 0: após 150 g; 0: após 200 g \\
\hline 7,8 e 9 & 200 & 300 & 1,5 & 0: após 50 g; 0: após $150 \mathrm{~g}$ \\
\hline
\end{tabular}


Levantamento das distribuições de temperatura em leito de arroz com casca durante o tratamento térmico por micro-ondas BERTELI, M. N. et al.

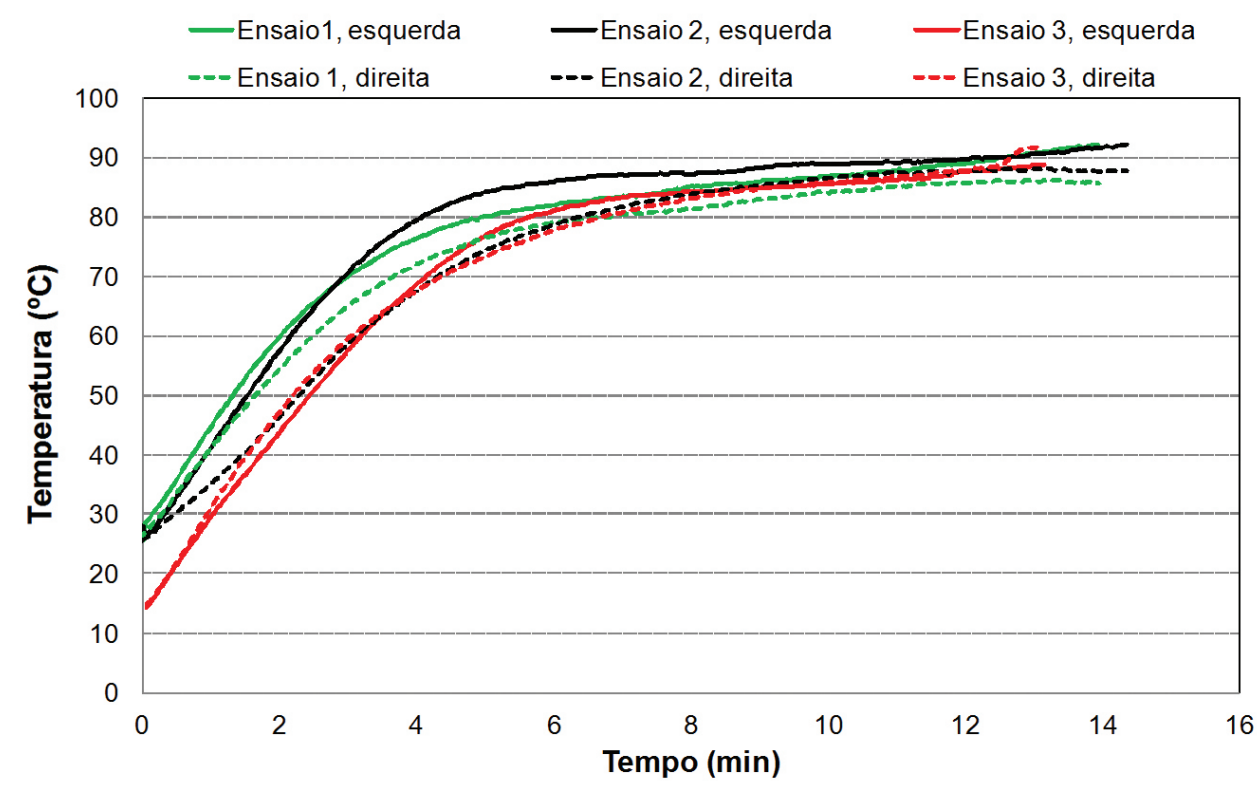

Figura 3. Evolução das temperaturas no leito de arroz referentes aos ensaios 1, 2 e 3; densidade de potência inicial: 1 W/g.

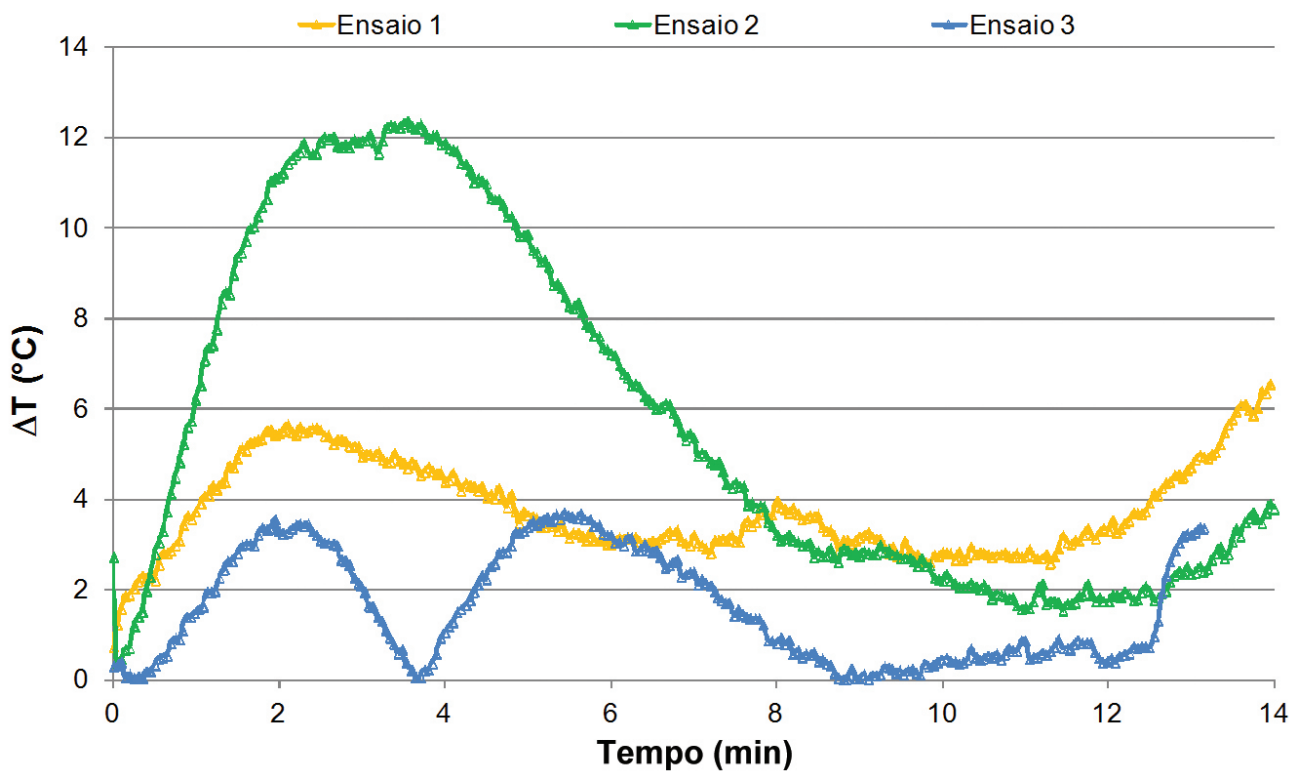

Figura 4. Evoluções das diferenças de temperatura registradas pelas fibras ópticas no leito de arroz referentes aos ensaios 1,2 e 3; densidade de potência inicial: $1 \mathrm{~W} / \mathrm{g}$.

foram realizados com as fibras posicionadas no lado esquerdo e no lado direito do cesto e com $\rho_{\mathrm{p}}=1,0 \mathrm{~W} / \mathrm{g}$.

A diferença máxima de temperatura $\left(\Delta T_{\max }\right)$ entre as duas fibras durante $\mathrm{o}$ ensaio 1 foi de $6,5^{\circ} \mathrm{C}$, no final do aquecimento, aos $14 \mathrm{~min}$, sendo que as maiores $\Delta \mathrm{T}$ entre os sensores foram de cerca de $5,0{ }^{\circ} \mathrm{C}$ a $5,6^{\circ} \mathrm{C}$, registradas entre 1,5 e 3,1 min e entre $5,0^{\circ} \mathrm{C}$ e $6,5^{\circ} \mathrm{C}$, dos 13,2 min aos 14 min (Figure 4). Observou-se ainda que a fibra posicionada no lado direito do cesto registrou durante todo o aquecimento temperaturas do arroz mais elevadas em relação às temperaturas registradas no lado esquerdo (Figura 3).

No ensaio 2, as $\Delta T$ entre as fibras ópticas ficaram próximas de $12^{\circ} \mathrm{C}$ dos $2,1 \mathrm{~min}$ aos $4,2 \mathrm{~min}$, ocorrendo a $\Delta \mathrm{T}_{\max }=12,3^{\circ} \mathrm{C}$ aos 3,5 min (Figura 4). O tempo necessário para que uma das fibras ópticas atingisse $92{ }^{\circ} \mathrm{C}$ também 
foi de 14 minutos. Nesse ensaio, a temperatura do produto posicionado à esquerda do cesto foi maior que a temperatura do produto posicionado à direita (Figura 3).

De acordo com os resultados observados no ensaio 3, as $\Delta T$ entre as fibras ópticas ficaram abaixo de 3,6 ${ }^{\circ} \mathrm{C}$, sendo esse valor máximo obtido aos 5,4 min (Figura 4). O final do aquecimento, quando a temperatura do produto atingiu $92^{\circ} \mathrm{C}$, ocorreu aos $13 \mathrm{~min}$. As maiores temperaturas oscilaram entre o lado esquerdo e direito (Figura 3).

A partir dos resultados dos ensaios 1, 2 e 3 não foi observado um comportamento padrão que indicasse uma região de maior aquecimento no leito de arroz na direção radial do cesto de Teflon.

As Figuras 5 e 6 apresentam os resultados obtidos nos ensaios 4, 5 e 6, com as fibras ópticas posicionas no centro do cesto de Teflon, a altura correspondente ao meio e à superfície do leito de arroz e com $\rho_{\mathrm{p}}=0,5 \mathrm{~W} / \mathrm{g}$. Durante os respectivos experimentos, o tempo necessário para que uma das fibras ópticas registrasse $92^{\circ} \mathrm{C}$ foi de 5,0 min a 6,0 min.

Observou-se que, nos ensaios 4 e 5 , as camadas centrais do leito de arroz registraram temperaturas mais elevadas em relação à da superfície. A situação se

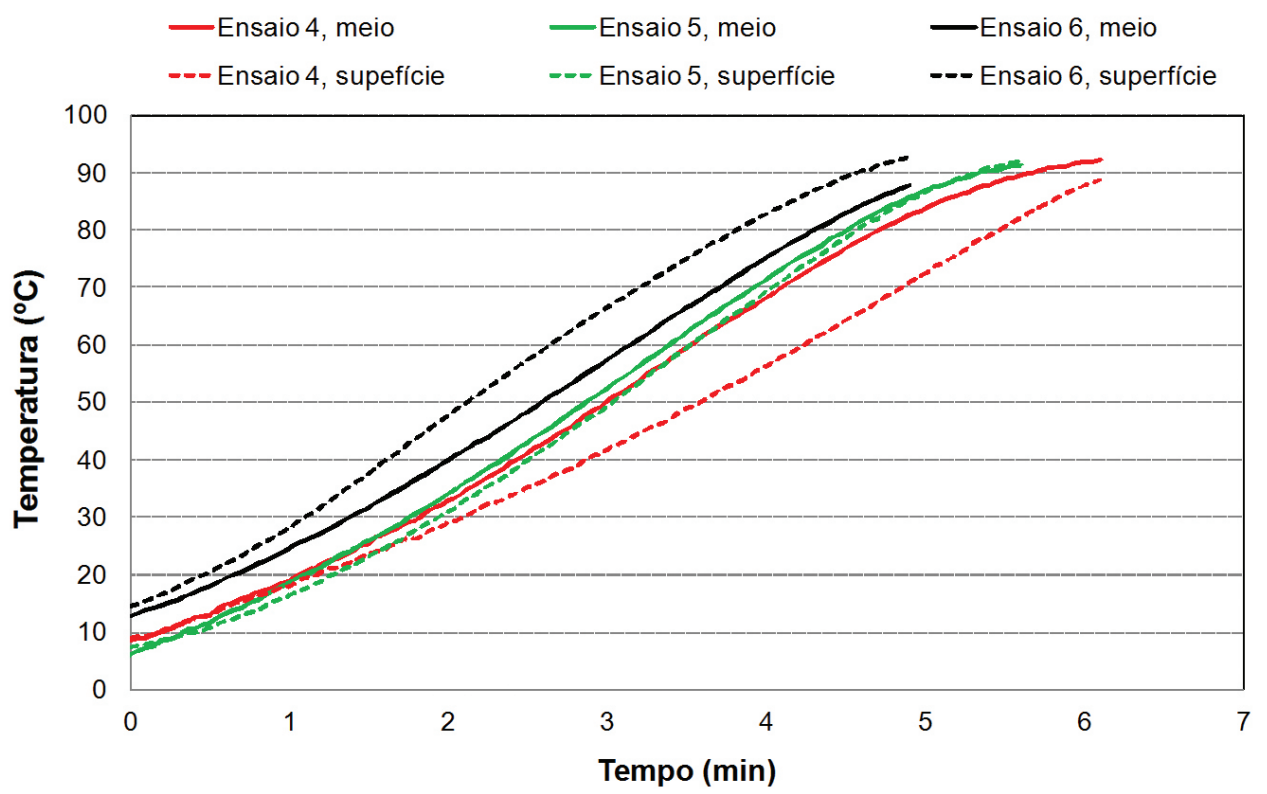

Figura 5. Evolução da temperatura do arroz nos ensaios 4, 5 e 6; densidade de potência inicial: 0,5 W/g.

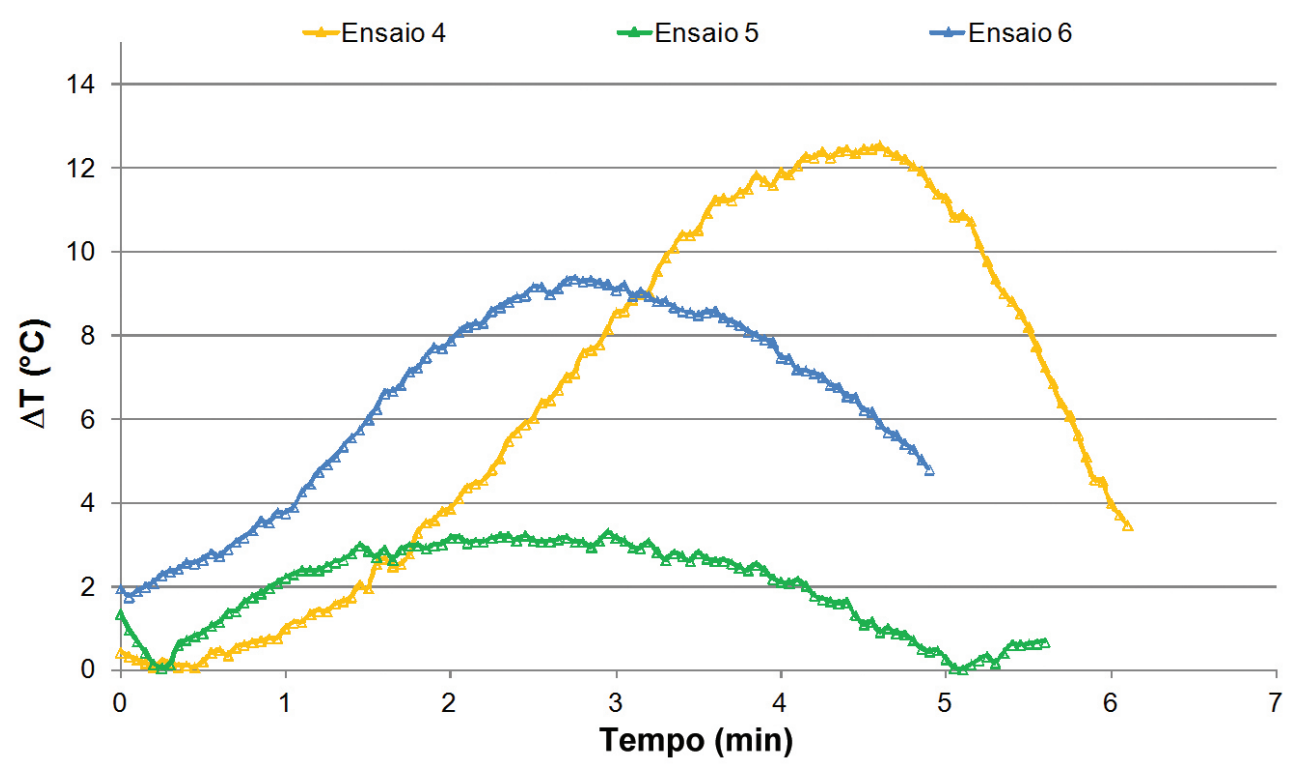

Figura 6. Evolução das diferenças na temperatura registrada pelas fibras ópticas no leito de arroz referentes aos ensaios 4,5 e 6; densidade de potência inicial: 0,5 W/g. 
Levantamento das distribuições de temperatura em leito de arroz com casca durante o tratamento térmico por micro-ondas BERTELI, M. N. et al.

inverteu no ensaio 6, onde as maiores temperaturas foram registradas na superfície do leito de arroz em relação ao seu centro (Figura 5).

No ensaio 4 , as $\Delta T$ entre as fibras ópticas ficaram acima de $10^{\circ} \mathrm{C}$ dos 3,3 min aos 5,2 min, atingindo a $\Delta \mathrm{T}_{\max }=12,5^{\circ} \mathrm{C}$ aos $4,6 \mathrm{~min}$. De acordo com os resultados do ensaio 5 , as $\Delta T$ entre as fibras ópticas ficaram abaixo de $3,2{ }^{\circ} \mathrm{C}$, sendo esse o valor máximo obtido, aos 2,9 min. Já no ensaio 6 , as $\Delta T$ entre as fibras ópticas ficaram acima de $8^{\circ} \mathrm{C}$ entre 2,0 min e 3,8 min, registrando-se a $\Delta \mathrm{T}_{\max }=9,3^{\circ} \mathrm{C}$ aos $2,7 \mathrm{~min}$ de processo.

Os resultados dos ensaios 7, 8 e 9, cujas temperaturas foram acompanhadas no fundo e no meio do leito de arroz, com $\rho_{\mathrm{P}}=1,5 \mathrm{~W} / \mathrm{g}$, estão apresentados nas Figuras 7 e 8 . Durante os experimentos, o tempo necessário para que uma das fibras ópticas registrasse $92{ }^{\circ} \mathrm{C}$ foi de 4,0 min a 4,5 min.

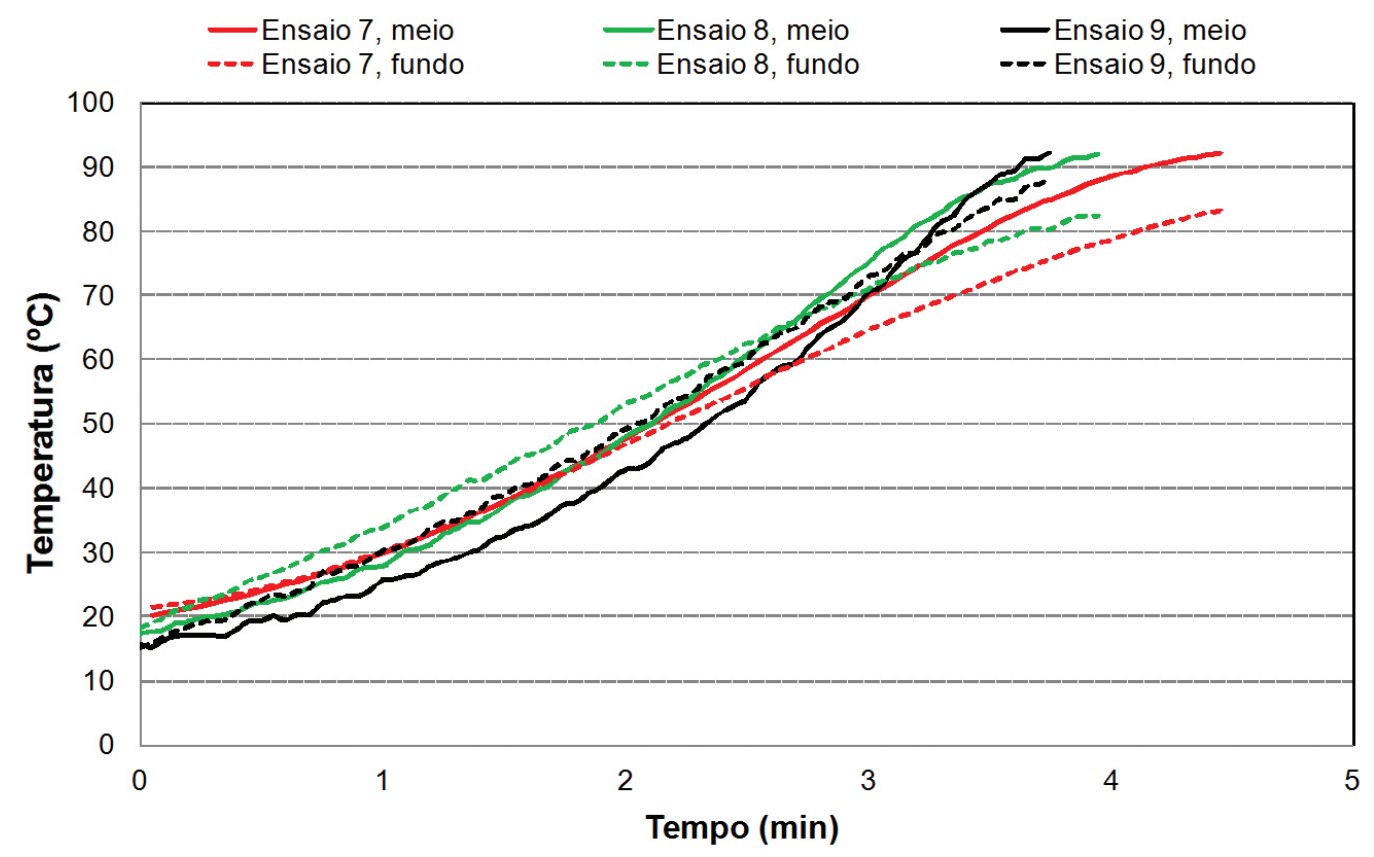

Figura 7. Evolução da temperatura do arroz nos ensaios 7, 8 e 9; densidade de potência inicial: 1,5 W/g.

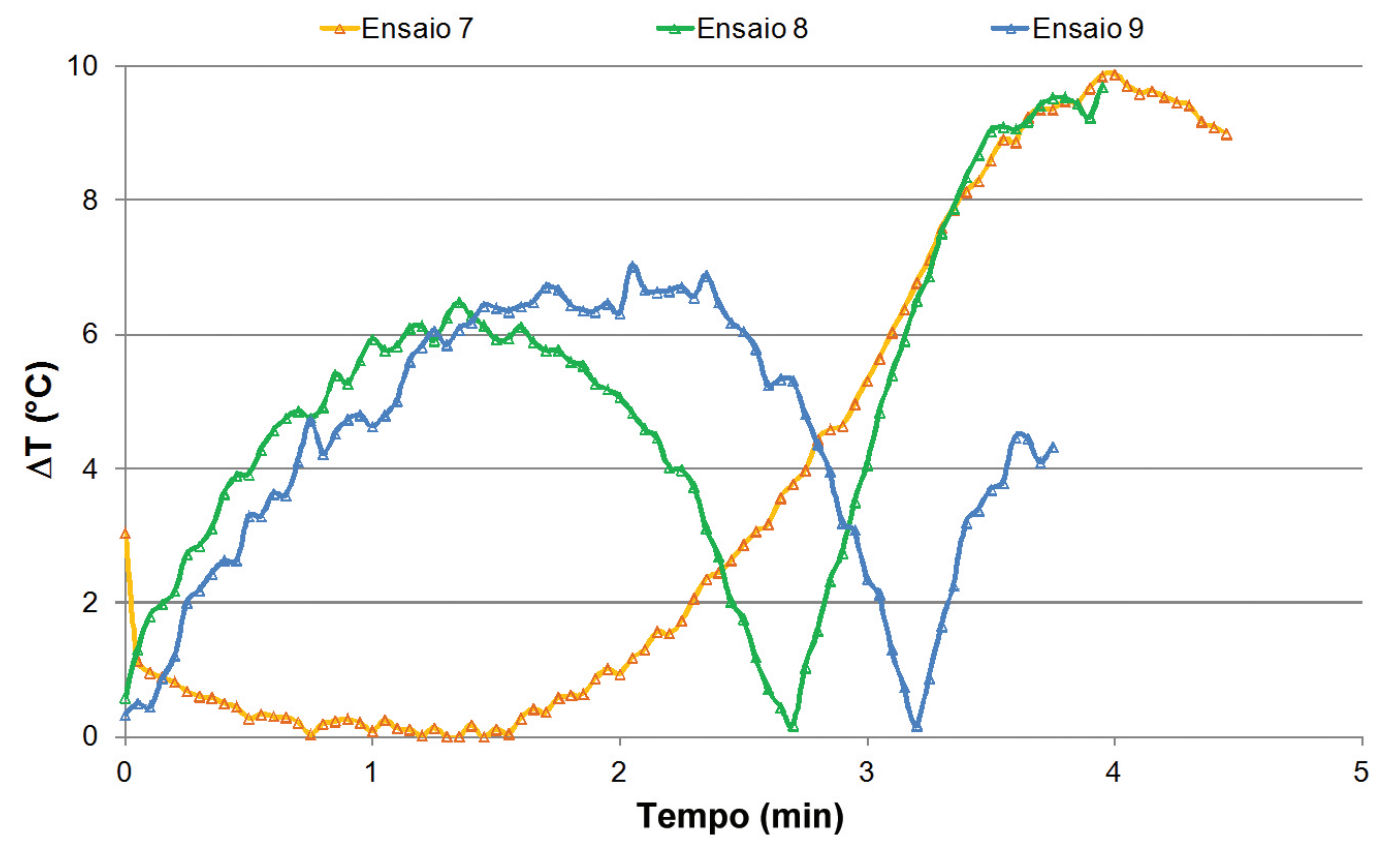

Figura 8. Evolução da diferença de temperatura registrada pelas fibras ópticas no leito de arroz referentes aos ensaios 7, 8 e 9; densidade de potência inicial: $1,5 \mathrm{~W} / \mathrm{g}$. 
Tabela 3. Resultados dos ensaios desenvolvidos no cesto de Teflon, sendo: $P_{T}=$ potência transmitida; $\rho_{p}=$ densidade de potência inicial; $U_{f}=$ umidade do produto em b.u. após o tratamento térmico; $\Delta T_{\max }=$ variação máxima de temperatura no processo entre as fibras ópticas; e tempo = tempo de processo térmico.

\begin{tabular}{|c|c|c|c|c|c|c|}
\hline Ensaio & $P_{T}(W)$ & $\rho_{p}(W / g)$ & $U_{f}(\%)$ & $\Delta \mathrm{T}_{\max }\left({ }^{\circ} \mathrm{C}\right)$ & Posição da fibra óptica & Tempo (min) \\
\hline 1 & 100 & 1,0 & 17,8 & 6,5 & E e D & 14,0 \\
\hline 2 & 100 & 1,0 & 16,7 & 12,3 & E e D & 14,0 \\
\hline 3 & 100 & 1,0 & 17,8 & 3,6 & E e D & 13,0 \\
\hline 4 & 100 & 0,5 & 26,9 & 12,5 & 0: centro/superfície & 6,1 \\
\hline 5 & 100 & 0,5 & 27,3 & 3,2 & 0: centro/superfície & 5,6 \\
\hline 6 & 100 & 0,5 & 27,0 & 9,3 & 0: centro/superfície & 4,9 \\
\hline 7 & 300 & 1,5 & 20,8 & 9,8 & 0: centro/fundo & 4,5 \\
\hline 8 & 300 & 1,5 & 25,7 & 9,7 & 0 : centro/fundo & 4,0 \\
\hline 9 & 300 & 1,5 & 24,4 & 7,0 & 0: centro/fundo & 3,7 \\
\hline
\end{tabular}

Observou-se no ensaio 7 que as camadas centrais do leito de arroz registraram temperaturas mais elevadas em relação às da superfície. O mesmo ocorreu no ensaio 8, entretanto, a partir de 2,6 min, a fibra óptica posicionada no fundo do leito de arroz passou a registrar temperaturas mais altas. A situação se inverteu no ensaio 9 , onde as maiores temperaturas foram registradas pela fibra posicionada no fundo do leito de arroz e, no final do aquecimento, pela posicionada no centro do produto.

A partir dos resultados obtidos pelos ensaios 4 a 9 , assim como ocorreu na direção radial, não foi observado um comportamento padrão que indicasse uma região de maior aquecimento no leito de arroz em relação à altura do leito no cesto de Teflon.

De acordo com a Figura 8 , no ensaio 7 as $\Delta T$ entre as fibras ópticas ficaram acima de $8{ }^{\circ} \mathrm{C}$ a partir dos 3,4 min, atingindo a $\Delta T_{\max }=9,8{ }^{\circ} \mathrm{C}$ aos $4,0 \mathrm{~min}$ de processo. No ensaio 8 , as $\Delta \mathrm{T}$ entre as fibras ópticas ficaram acima de $8{ }^{\circ} \mathrm{C}$ no final do processo térmico, a partir dos $3,4 \mathrm{~min}$, atingindo a $\Delta \mathrm{T}_{\max }=9,7^{\circ} \mathrm{C}$ aos $4,0 \mathrm{~min}$. O ensaio 9 apresentou a $\Delta \mathrm{T}_{\max }=7,0^{\circ} \mathrm{C}$, aos $2,0 \mathrm{~min}$, e $\Delta \mathrm{T}$ acima de $6{ }^{\circ} \mathrm{C}$ de 1,3 a 2,5 min.

A Tabela 3 apresenta o resumo dos principais resultados obtidos durante o tratamento do arroz com casca com micro-ondas dentro da cavidade prismática. Após cada tratamento térmico foi determinada a umidade final da amostra em um analisador de umidade por microondas (Smart 5 - CEM).

Observa-se, de acordo com a Tabela 3, que para os menores valores de densidade de potência inicial, $\rho_{\mathrm{p}}=0,5 \mathrm{~W} / \mathrm{g}$, foram obtidos produtos com maiores valores de umidade final, referentes aos testes 4,5 e 6 .

Os ensaios 1,2 e 3 , realizados com $\rho_{p}=1 \mathrm{~W} / \mathrm{g} \mathrm{e}$ com a menor massa de material, $M=100 \mathrm{~g}$, foram os que apresentaram os maiores tempos de processo, o que resultou em um produto final com menor umidade final.
Os ensaios 7, 8 e 9, cujo valor de $P_{T}$ foi o maior, $300 \mathrm{~W}$, foram os que apresentaram menores tempos de processo e os testes 7 e 8 , as maiores $\Delta T$ no final de cada processo.

A partir dos resultados observa-se que a diferença de temperatura do produto na cavidade multimodo fixa durante o tratamento de arroz em casca por micro-ondas foram de $3,2^{\circ} \mathrm{C}$ a $12,5^{\circ} \mathrm{C}$.

Ainda que a cavidade avaliada neste estudo apresente geometria que minimiza a heterogeneidade do campo elétrico, durante o aquecimento dielétrico outros fatores afetaram o aquecimento do produto, o que pode ter influenciado a variação de temperatura (WÄPPLINGRAAHOLT e OHLSSON, 2005). A absorção da potência e a intensidade do campo no interior dos materiais variaram com a profundidade de penetração das micro-ondas. Essas condições variam também com a temperatura durante o aquecimento por micro-ondas. Desse modo, o fator perda dielétrica relativa $\varepsilon^{\prime \prime}$ varia com o tempo e de uma região à outra do material. Além desses fatores citados, inclui-se a própria variação da composição do material e a alteração da distribuição do campo elétrico quando um material dielétrico é inserido na cavidade.

\section{Conclusões}

As diferenças de temperatura observadas durante os tratamentos de arroz em casca na cavidade prismática multimodo fixa foram de $3,2^{\circ} \mathrm{C}$ a $12,5^{\circ} \mathrm{C}$.

Não foi observado um comportamento padrão que indicasse uma região de maior aquecimento em relação à altura e na direção radial do leito de arroz posicionado no cesto de Teflon dentro da cavidade durante os tratamentos térmicos por micro-ondas estudados.

\section{Agradecimentos}

Os autores agradecem ao CNPq pelo financiamento do presente estudo. 
Levantamento das distribuições de temperatura em leito de arroz com casca durante o tratamento térmico por micro-ondas BERTELI, M. N. et al.

\section{Referências}

ASSOCIATION OF OFFICIAL ANALYTICAL CHEMIST - AOAC. Guidelines for laboratories performing microbiological and chemical analyses of food and pharmaceuticals. Rockville, MD: AOAC, 2006.

BOLDOR, D.; BALASUBRAMANIAN, S.; PUROHIT S.; RUSCH K. A. Design and implementation of a continuous microwave heating system for ballast water treatment. Environmental Science and Technology, Columbus, v. 42, n. 11, p. 4121-4127, 2008. http://dx.doi.org/10.1021/es7024752

BUFFLER, C. R. Microwave cooking and processing: engineering fundamentals for the food scientist. New York: V. N. Reinhold AVI, 1992. 169 p.

CORONEL, P.; SIMUNOVIC, J.; SANDEEP, K. P.; CARTWRIGHT, G. D.; KUMAR, P. Sterilization solutions for aseptic processing using a continuous flow microwave system. Journal of Food Engineering, Amsterdam, v. 85, n. 4, p. 528-536, 2008. http:// dx.doi.org/10.1016/j.jfoodeng.2007.08.016

KOMAROV, V. Foodstuff and agricultural products. In: KOMAROV, $\checkmark$. Handbook of dielectric and thermal properties of material at microwave frequencies. Boston: Artech House, 2012. cap. 3, p. 29-68.

KOSKINIEMI, C. B.; TRUONG, V.-D.; SIMUNOVIC, J.; McFEETERS, R. F. Improvement of heating uniformity in packaged acidified vegetables pasteurized with a $915 \mathrm{MHz}$ continuous microwave system. Journal of Food Technology, Paquistão, v. 105, n. 1, p. 149-160, 2011. http://dx.doi. org/10.1016/j.jfoodeng.2011.02.019

KRASZEWSKI, A.; NELSON, S. O. Composite model of the complex permittivity of cereal grain. Journal of Agricultural Engineering Research, Bedford, v. 43, p. 211-219, 1989.

LIN, M.; RAMASWAMY, H. S. Evaluation of phosphatase inactivation kinectics and conventional heating conditions.
International Journal of Food Properties, London, v. 14, $\mathrm{n}$. 1, p. 110-123, 2011.

NELSON, S. O.; DATTA, A. K. Dielectric properties of food materials and electric field interactions. In: DATTA, A. K.; ANANTHESWARAN, R. C. Handbook of microwave technology for food applications. New York: Marcel Dekker, 2001. cap. 3, p. 69-114.

RAYAGURU, K.; PANDEYJ. P.; ROUTRAY, W. Optimization of process variables for accelerated aging of basmati rice. Journal of Food Quality, Malden, v. 34, n. 1, p. 56-63, 2011. http://dx.doi. org/10.1111/j.1745-4557.2010.00366.x.

RISMAN, P. Advanced topics in microwave heating uniformity. In: LORENCE, M.; PESHECK, P. S. Development of packaging and products for use in microwave ovens. Boca Raton: $C R C$ Press, 2009. cap. 3, p. 66-104.

ROCHA, C. R. Efeitos do tratamento por microondas do arroz recém colhido no rendimento de grãos inteiros, na qualidade de cozimento e na estabilização do farelo. 2002. $160 \mathrm{f}$. Tese (Doutorado em Engenharia de Alimentos)-Faculdade de Engenharia de Alimentos, Universidade Estadual de Campinas, Campinas, 2002.

TANG, J. Dielectric properties of foods. In: SCUBERT H.; REGIER, M (Eds.). The microwave processing of foods. Boca Raton: CRC Press, 2005. cap. 2, p. 22-40.

VADIVAMBAL, R.; JAYAS, D. S. Non-uniform temperature distribution during microwave heating of food materials: a review. Food and Bioprocess Technology, New York, v. 3, n. 2, p. 161-171, 2010. http://dx.doi.org/10.1007/s11947-008-0136-0.

WÄPPLING-RAAHOLT, B.; OHLSSON, T. Improving the heating uniformity in microwave processing. In: SCHUBERT, H.; REGIER, $M$ (Eds.). The microwave processing of foods. Boca Raton: CRC Press, 2005. cap. 15, p. 292-316. 